\title{
Negative and positive regulation of Tn10/IS10-promoted recombination by IHF: two distinguishable processes inhibit transposition off of multicopy plasmid replicons and activate chromosomal events that favor evolution of new transposons
}

\author{
Laurence Signon $^{1}$ and Nancy Kleckner ${ }^{2}$ \\ Department of Biochemistry and Molecular Biology, Harvard University, Cambridge, Massachusetts 02138 USA
}

Tn10 is a composite transposon; inverted repeats of insertion sequence IS10 flank a tetracycline-resistance determinant. Previous work has identified several regulatory processes that modulate the interaction between Tn10 and its host. Among these, host-specified DNA adenine methylation, an IS10-encoded antisense RNA and preferential cis action of transposase are particularly important. We now find that the accessory host protein IHF and the sequences that encode the IHF-binding site in IS10 are also important regulators of the Tn10 transposition reaction in vivo and that these determinants are involved in two distinguishable regulatory processes. First, IHF and the IHF-binding site of IS10, together with other host components (e.g., HU), negatively regulate the normal intermolecular transposition process. Such negative regulation is prominent only for elements present on multicopy plasmid replicons. This multicopy plasmid-specific regulation involves effects both on the transposition reaction per se and on transposase gene expression. Second, specific interaction of IHF with its binding site stimulates transposon-promoted chromosome rearrangements but not transposition of a short Tn10-length chromosomal element. However, additional considerations predict that IHF action should favor chromosomal transposition for very long composite elements. On the basis of these and other observations we propose that, for chromosomal events, the major role of IHF is to promote the evolution of new IS10-based composite transposons.

[Key Words: IHF; Tn10; transposon; evolution; plasmid; chromosome rearrangements]

Received December 19, 1994; revised version accepted March 20, 1995.

Tn10 is a composite transposon; it comprises IS10 insertion sequences that flank tetracycline-resistance genes (Fig. 1; Kleckner 1989). IS10 is an independent transposition module that encodes a single transposase protein. The two ends of IS10 share a nearly perfect terminal inverted repeat, the transposase-binding site but are genetically distinguished as a consequence of base-pair differences within that site and by the presence of a binding site for $E$. coli integration host factor (IHF) adjacent to the outside end-terminal inverted repeat (Fig. 1; Huisman et al. 1989).

Tn10 and IS10 transpose nonreplicatively. The ele-

\footnotetext{
'Present address: Department of Biology, Brandeis University, Waltham, Massachusetts USA.

${ }^{2}$ Corresponding author.
}

ment is excised from the donor site and inserted into a new target site (Bender and Kleckner 1986). The two chromosome ends left behind by $\mathrm{Tn} 10$ transposon excision are not directly rejoined (Bender et al. 1991). The donor chromosome is presumably either degraded and/ or repaired by a recombinational mechanism with information from a sister chromosome (Bender and Kleckner 1986; Bender et al. 1991). The transposition reaction can be subdivided into four successive stages, each dependent on the one before: (1) formation of a synaptic complex between the transposon ends; (2) double-strand cleavage at the two ends of the element; $(3)$ noncovalent capture of a target DNA; and (4) strand transfer of transposon ends to target DNA (Haniford et al. 1991; Benjamin and Kleckner 1992; Chalmers and Kleckner 1994; J. Sakai and N. Kleckner, in prep.). 


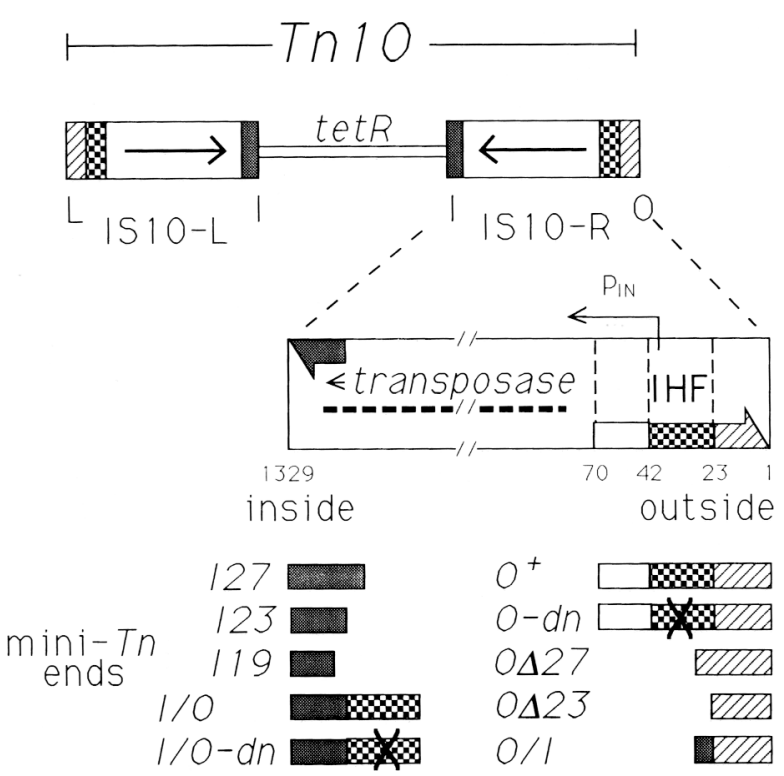

Figure 1. Structures of $\operatorname{Tn} 10$, IS10, and relevant minitransposon ends. $\mathrm{O}^{+}$consists of wild-type IS10 base pairs 1-70 (Halling et al. 1982); sequences adjacent to base pair 1 correspond to those adjacent to IS10-Right in hisG9424::Tn10 (Halling and Kleckner 1982); sequences beyond base pair 70 are specific linker base pairs. $\mathrm{O}-d n$ is $\mathrm{O}^{+}$with three AT> TA transversions at three consensus base pairs in the IHF-binding site (Huisman et al. 1989); $O D 27$ is $\mathrm{O}^{+}$with a deletion from base pairs 28-70; $O \Delta 23$ is the same as $O \Delta 27$ except that the deletion extends from base pairs $24-70 ; O / I$ is the same as $O \Delta 27$ except that base pairs 24-27 are those found at the corresponding position in the inverted repeat at the inside end of IS10,127, 123, and 119are the same as $\mathrm{O}^{+}$except that the only transposon sequences present are the first 27,23, and $19 \mathrm{bp}$ from the inside end of IS10-Right (base pairs 1329-1303, 1307, and 1311, respectively); $1 / O$ and $I / O-d n$ are the same as $\mathrm{O}^{+}$and $O-d n$ except that the first $23 \mathrm{bp}$ of the inside end (base pairs 1329-1307) have been substituted for outside-end base pairs 1-23. The outside end of IS10-Left is slightly different in sequence from the outside end of IS10-Right and is thus designated $\mathrm{L}$ rather than $\mathrm{O}$.

Tn10 and IS10 not only undergo transposition but also promote chromosome rearrangements. Tn10 promotes both deletion and inversion of adjacent flanking sequences by events in which the two internal ends of the element interact with an adjacent target site in a random collision process (Fig. 2; Kleckner et al. 1979; Benjamin and Kleckner 1992). Information originally located between the inside ends is lost and a segment extending from one inside end to an adjacent target site is inverted, such that a pair of IS10 elements oriented as direct repeats now flank the previously adjacent chromosomal segment. Inversion events promoted in this way by pairs of IS sequences are of particular biological importance because they generate composite transposable elements encoding new genetic determinants (Fig. 2; Wolf 1980; Foster et al. 1981; Raleigh and Kleckner 1984; Navas et al. 1985; Roberts 1986).

Many types of IS elements and their composite trans- posons appear to be evolutionarily successful, stable components of bacterial genomes (e.g., Lawrence et al. 1989; Bisercic and Ochman 1993; Romero and Klaenhammer 1993; Stanley et al. 1993). The perpetuation of such a relationship requires that effects of transposition deleterious to the host be minimized while sufficient transposition activity is maintained to ensure maintenance of the transposable element. Tn10 and IS10 transposition are known to be subject to regulation or modulation in a number of different ways that can be rationalized on the basis of their advantages for the transposon/host relationship (for review, see Kleckner $1990 \mathrm{a}, \mathrm{b})$.

Here, we address the biological roles of the accessory host factor IHF in Tn10/IS10 activity in vivo. An IHFbinding site consensus sequence of moderate strength occurs immediately adjacent to the transposase-binding site at the outside end of IS10 (Fig. 1; Huisman et al. 1989|. Previous work suggested that IHF can affect both transposase expression and the transposition reaction per se but the exact nature of these roles has been unclear (Roberts 1986; Roberts et al. 1987; Morisato and Kleckner 1987; Huisman et al. 1989; D. Morisato, H. Benjamin, and R. Chalmers, unpubl.).

IHF and its relative host component (HU) are small DNA-binding proteins that mediate changes in the structure of DNA. IHF binds tightly to a specific consensus sequence, making a $140^{\circ}$ bend in the DNA /Yang and Nash 1989; Nash 1990). HU binds weakly and relatively nonspecifically to DNA but prefers sequences that are naturally bent or flexible (Broyles and Pettijohn 1986;

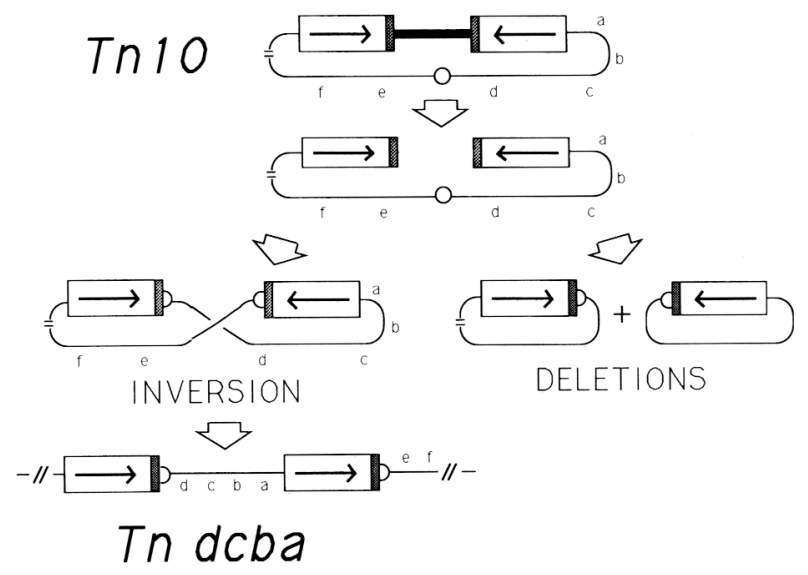

Figure 2. Chromosome rearrangements promoted by $\operatorname{Tn} 10$. Any composite IS-based transposon will generate both deletions and inversions as alternative reaction products. Each inversion event generates a new composite transposon, e.g., Tn- $d c b a$. It should be noted that the presence of two IS elements effectively bisects the chromosome into two transposons: Tn10, and a transposon that comprises the rest of the bacterial chromosome. With respect to this latter transposon therefore, Tn10promoted deletions and inversions are intra-transposon events, i.e., events in which the target site is located within the transposon itself. 
Pettijohn and Hodges-Garcia 1990; Pontiggia et al. 1993; Bonnefoy et al. 1994).

In several site-specific recombination and transposition reactions, IHF and $\mathrm{HU}$ are known to play important architectural roles (e.g., Nash 1990; Baker and Mizuuchi 1992; Haykinson and Johnson 1993; Lavoie and Chaconas 1993; Segall et al. 1994). In each of these cases, biochemical processes have been placed under host factor control to permit their regulation by other components. The complex architecture of the $\lambda$ attachment site permits differential regulation of integration and excision; for Hin inversion, HU helps to favor the formation of inversion events over deletions, and the IHF-dependent step in assembly of the Mu synaptic complex permits regulation of transposition by repressor and ensures that $\mathrm{Mu}$ never inserts into itself. In vivo and in vitro observations suggest that $\mathrm{HU}$ can substitute for IHF as an architectural element (e.g., Segall et al. 1994 and references therein).

The experiments described below investigate the biological roles of IHF and the sequences that encode the IHF-binding site at the outside end of IS10 during Tn10promoted recombination in vivo. Intermolecular transposition both from the chromosome and from a plasmid donor, and Tn10-promoted chromosomal rearrangements have all been assayed in $\mathrm{IHF}^{+}$and $\mathrm{IHF}^{-}$strains. To permit analysis of changes in the transposition process per se, transposase has been provided at varying levels from a heterologous promoter. Such analyses have been carried out with minitransposons comprising a variety of wild-type and mutant IS10 ends.

The effects of IHF on transposase gene expression are also considered briefly. When IS10 is present on a multicopy plasmid, interaction of IHF at the IHF-binding site inhibits transposase expression at the transcriptional level, presumably by affecting the overlapping -35 region of the transposase gene promoter $p I N$ (Huisman et al. 1989; Sussman 1992; Ditto et al. 1994). Results presented below suggest that this inhibition of transposase gene expression does not occur for IS10 elements in the chromosome.

\section{Results}

Assays for transposition and chromosome rearrangements

Endogenous intermolecular transposition of Tn10 has been assayed by a gene activation assay in which transposition results in expression of a suitably positioned kan gene lacking transcription and translational start signals (Bender et al. 1991). A 'kan ery minitransposon was used (Fig. 3A). The identical constructs, together with adjacent flanking sequences, were examined both on a multicopy pBR322-based plasmid replicon and in the chromosome via isogenic single copy $\lambda$ prophages (Materials and methods).

Chromosome rearrangements have been monitored by a newly developed version of the classical assay. A pair of transposon ends oriented to mimic the inner ends of
Tn10 flank a pair of markers whose loss can be selected (Fig. 3B). The transposon ends in such constructs, as well as the immediately flanking adjacent sequences, are exactly the kan and ery halves of the mini-transposon used for transposition assays. Thus, in so far as is possible, chromosome rearrangements have been assayed with constructs that are genetically identical to those used to monitor transposition. The intervening markers are a $s a c B$ gene, which confers sensitivity to sucrose (Gay et al. 1985) and a $\lambda \mathrm{cI}$ repressor gene, which confers sensitivity to tetracycline via a repressor-sensitive $P_{\mathrm{L}}-$ tet reporter fusion present on a separate replicon. The frequency of Tn10-promoted rearrangements is determined by selection for resistance to sucrose and/or tetracycline. Among such derivatives, the proportions of deletions and inversions are determined by scoring for the presence of the flanking 'kan and ery markers (Fig. 3B; Materials and methods).

In most experiments transposase was provided by a Ptac-transposase fusion present on a different replicon from the transposon construct, a modest copy number plasmid in the presence of a single chromosomal copy of the lacI repressor gene. The effective transposase concentration present in this standard condition is in the same range as, but probably severalfold higher than, that seen by wild-type IS10 making its own transposase (Way 1984). In some experiments higher or lower effective levels of transposase were examined, and one experiment compares expression from Ptac with expression from the wild-type transposase gene promoter, $P-I N$.

Transposition and chromosome rearrangements have both been examined in several different strain backgrounds. Results are reported here primarily for two strain backgrounds, NK7419 and NK8087 and appropriate isogenic $\mathrm{IHF}^{-}$derivatives; in one set of experiments, two additional strain backgrounds were examined, NK7814 and DRC325 and their IHF derivatives (Materials and methods).

$\operatorname{Tn} 10$ activity was examined in most detail for constructs comprising two IS10-Right outside ends. The activity of wild-type outside ends $\left(\mathrm{O}^{+}\right)$was compared with the activity of several altered ends compromised with respect to the IHF-binding site (Fig. 1). Mutant ends either carry an inactivating point mutation in the IHFbinding site $(O-d n)$ or are deleted for the IHF-binding site $(O \Delta 27$ and $O \Delta 23 \mid$, and a hybrid end carries a few insideend base pairs in place of the IHF-binding site $(O / I)$. Some experiments examined isogenic constructs comprising two inside ends $(I 27, I 23$, or $I 19)$ or hybrid ends consisting of the inside end-terminal inverted repeat plus adjacent end sequences encoding the IHF-binding site $(I / O$ and the mutant derivative $I / O-d n)$.

In general, for each activity assay, the relative activities observed for the four genetic situations analyzed, wild-type end, and any particular mutant end in both $\mathrm{IHF}^{+}$and $\mathrm{IHF}^{-}$hosts, are presented as a rectangular array (Fig. 4A). The wild-type and doubly defective cases occupy the upper left and lower right corners of the rectangle and the singly defective cases occupy the other two corners. The ratio of the frequencies observed for five 
Figure 3. Transposon activity assays. (A) Transposition of a mini $\operatorname{Tn} 10$-kan ery $R$ transposon from an appropriate donor site to an appropriate new insertion site confers resistance to kanamycin (Bender et al. 1991). The 'kan segment comprises a nearly complete coding sequence lacking transcriptional and translational start signals. This segment is positioned with the proximal end of the gene located near one end of the transposon and adjacent terminal sequences are devoid of stop codons in-frame to the ' $k a n$-coding sequence. Insertion of this element into an actively expressed gene in appropriate orientation and reading frame will result in expression of the kan polypeptide. Insertions $(1 / 500-1 / 1000)$ of this element into the $E$. coli chromosome confer kanamycin resistance (J. Mahillon and N. Kleckner, unpubl.). (B) Chromosome rearrangements are promoted by a pair of transposon ends oriented to mimic the innermost ends of a composite transposon, and the frequency of such rearrangements can be measured by determinination of the frequency with which the segment between those ends is lost (Kleckner et al. 1979). In the construct shown, the intervening segment confers sensitivity to both sucrose and tetracycline, and rearrangement frequency is determined by selection for resistance to one or both agents (Materials and methods|. Deletions and inversion are distinguished from one another by the presence or absence of erythromycin resistance, monitored phenotypically, and/or the 'kan segment, monitored by colony hybridization.

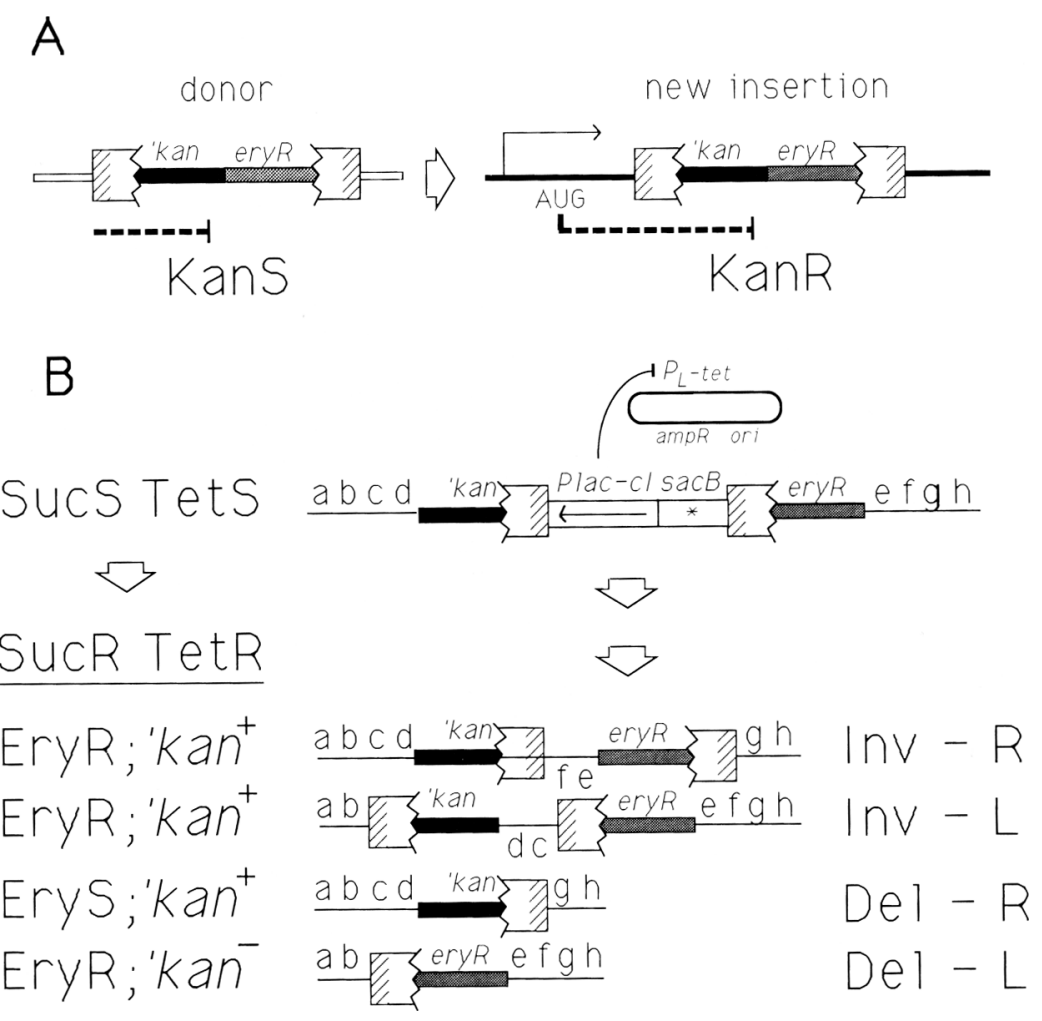

pertinent two-way comparisons are given along the connecting lines, with the arrowhead on each line pointing towards the combination that gave the higher frequency.

In each array the total effect of eliminating both IHF and the IHF-binding site is given by the direction and magnitude of the effect shown on the diagonal, that is, the comparison between the fully wild-type and doubly defective cases. The top horizontal and left-most vertical comparisons describe the consequences of eliminating either determinant singly, and the bottom horizontal and right-most vertical comparisons reveals the consequences of eliminating the second determinant when the first determinant has already been eliminated.

\section{Transposition of an outside-end minitransposon off of a multicopy plasmid increases in the absence of both IHF and the IS10 HF-binding site}

At the standard transposase level in NK7419, elimination of both IHF and the IS10 IHF-binding site results in an increase of $\sim 30$-fold in the level of Tn10 transposition from a multicopy plasmid (Fig. 4B). The same effect is observed with both $\mathrm{O}-d n$ and $\mathrm{O} / \mathrm{I}$ ends. The occurrence of such an increase was unexpected. It implies that transposition is normally subject to inhibitory effects that are dependent upon the two relevant determinants. We interpret these observations to mean that IHF and its cog- nate binding site participate in some type of biologically significant negative regulation.

\section{Negative regulation of plasmid transposition involves multiple determinants}

Negative regulation of plasmid transposition involves additional complexities. Transposition of outside end elements increases only when IHF activity and the IHFbinding site are both compromised (Fig. 4B). Elimination of IHF function causes only a marginal (approximately twofold) increase in transposition of a wild-type element; and in a wild-type strain, elimination of the IHFbinding sitc by point mutation or by deletion has essentially no effect. Thus, negative regulation involves one or more factors other than specific binding of IHF to its site. The observed pattern of effects can be explained by the supposition that HU can substitute for IHF in the presence of a wild-type IHF-binding site but not when the IHF-binding site is compromised and that IHF either still mediates negative regulation in the absence of an IHF-binding site (not such an unlikely possibility; see Discussion) or can act indirectly to promote reguation by $\mathrm{HU}$.

Furthermore, a very low transposase level appears to sensitize the transposition reaction to negative regulatory processes (Fig. 5). At transposase levels ranging from 
A

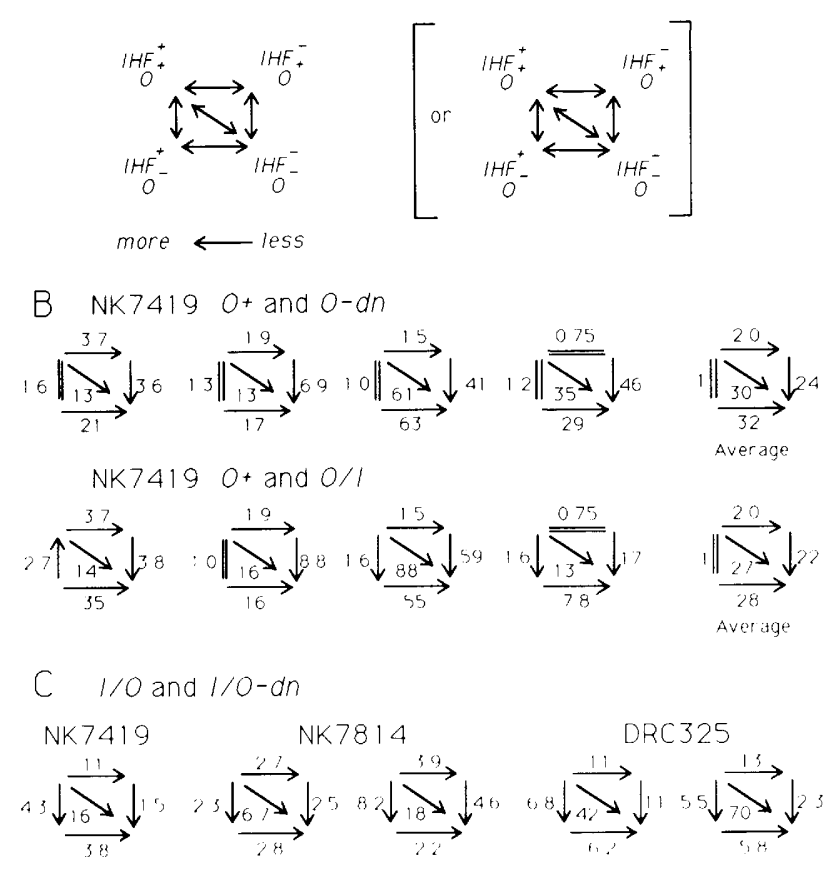

Figure 4. Intermolecular transposition from a multicopy plasmid. (A) Convention for presentation of data. Relative activities of a wildtype outside end $\left(\mathrm{O}^{+}\right)$and any given variant outside end in isogenic $\mathrm{IHF}^{+}$and $\mathrm{IHF}^{-}$strains are measured. For each such set of comparisons, five numbers are presented in the diagramed rectangular array. The five values given are the quotients of the frequencies obtained in the five corresponding pairs of situations. The arrow points towards the higher number of the pair. Of particular interest is the number present on the diagonal, which is the ratio of the frequencies obtained in the fully wild-type and doubly defective cases, and reveals the consequences of eliminating both IHF and the IHF-binding site in IS10. Numbers on the horizontal and vertical axes represent the effects of eliminating the two determinants sequentially in the two possible orders. The left-most pair represents the effects of each single defect in the presence of the other determinant, and the right-most pair represents the effects of eliminating the second determinant after the first determinant has already been eliminated. In principle, the number along the diagonal should be the same as the product of each pair of horizontal and vertical numbers. When this is not the case, the differences arise from the rounding off of component numbers in the latter comparisons. The averaged data from two or more experiments represents the averages of the five comparison numbers calculated for the component experiments. In cases where the comparison number fluctuates around the number one, the average is presented as $1 .(B)$ Intermolecular transposition was assayed for isogenic mini-Tn10-'kan ery $R$ plasmid constructs having pairs of identical $\mathrm{O}^{+}, \mathrm{O}-\mathrm{dn}$ or $\mathrm{O} / \mathrm{I}$ ends (pNK2824, pNK3344, pNK3346) were examined in isogenic $\mathrm{IHF}^{+}$

and IHF strains (NK7419 and NK8084) that also harbored a Ptac-transposase plasmid pNK3358. The Ptac promoter is probably not fully induced in these experiments; the host strain is lacI ${ }^{+}$. No external inducing agent (lactose or IPTG) was present at any point. Data are presented for four different experiments and the average of the four. Data for each experiment represent the average of three to five independent clones (Materials and methods). The absolute frequencies of kanamycin-resistant derivatives observed for the wild-type transposon in the $\mathrm{IHF}^{+}$strain (the upper left corner of each diagram) for the four experiments were, from left to right, $2 \times 10^{5}, 2 \times 10^{5}, 1 \times 10^{5}$, and $2 \times 10^{5}$. The background frequencies of kanamycin resistant derivatives observed for the wild-type transposon in the IHF ' and IHF strains in the absence of transposase, i.e., in the presence of an isogenic Tase " plasmid pNK2824 were always $<10^{9}$, well below the lowest levels observed in the presence of transposase. $(C)$ Comparison of $I / O$ and $I / O-d n$ ends in NK7419, NK7814, and DRC325 (and their isogenic IHF derivatives NK8084, NK7817, and DRC324). In these experiments, transposase was provided in cis by an insertion of the Ptac-transposase fusion into the plasmids carrying the ends $(I / O=\mathrm{pNK} 2820$; $I / O-d n=$ pNK2816). This results in a much higher effective level of transposase because transposase is preferentially cis acting (Morisato et al. 1983; Jain and Kleckner 1993), but the presence of a higher level does not significantly affect the effects observed, at least for outside end elements (Fig. 6). Frequencies of kanamycin-resistant derivatives observed for the I/O transposon in the $\mathrm{IHF}^{+}$ strains were (left to right): $4.6 \times 10^{-6}, 4.6 \times 10^{5}, 1.8 \times 10^{4}, 4.3 \times 10^{6}{ }^{6}$, and $5 \times 10^{-6}$; background observed in the absence of transposase (pNK2824) was $<10^{-9}$.

0.1 to 30 times the standard level, the observed increases in transposition are similar in each of the two strain backgrounds examined. But at a very low transposase level, elimination of IHF and the IHF-binding site causes an exaggeration in the increase in transposition. At 0.01 times the standard level of transposase, transposition increases $\sim 100$ - and $\sim 1000$-fold, respectively, in the two strain backgrounds.

\section{IS10 inside ends are also subject to negative regulation}

IHF-mediated negative regulation of plasmid transposition is also observed with minitransposon constructs comprising inside IS10 ends. For I27, I23, and I19 ends, which naturally lack any IHF-binding site, transposition in an $\mathrm{IHF}^{-}$strain is increased 5- to 20 -fold as compared with $\mathrm{IHF}^{+}$(Table 1). Moreover, addition of an IHF-bind- ing site to these $I^{+}$ends creates ends that are even more responsive to negative regulation than were the original $\mathrm{O}^{+}$ends. Elimination of IHF, in combination with a mutation in the IHF-binding site, results in a 70 -fold increase in transposition (Fig. 4C).

\section{Transposition of outside-end elements off of the bacterial chromosome is affected only marginally by negative regulation}

The prominent negative regulation observed on multicopy plasmids is minimal but discernible for transposition from the chromosome. At the standard transposase level in the NK7419 strain background used most extensively for plasmid assays, elimination of IHF and alteration of the IHF-binding site in combination cause chromosomal transposition to increase about twofold (Fig. 6). This modest increase is real. A similar increase has been 


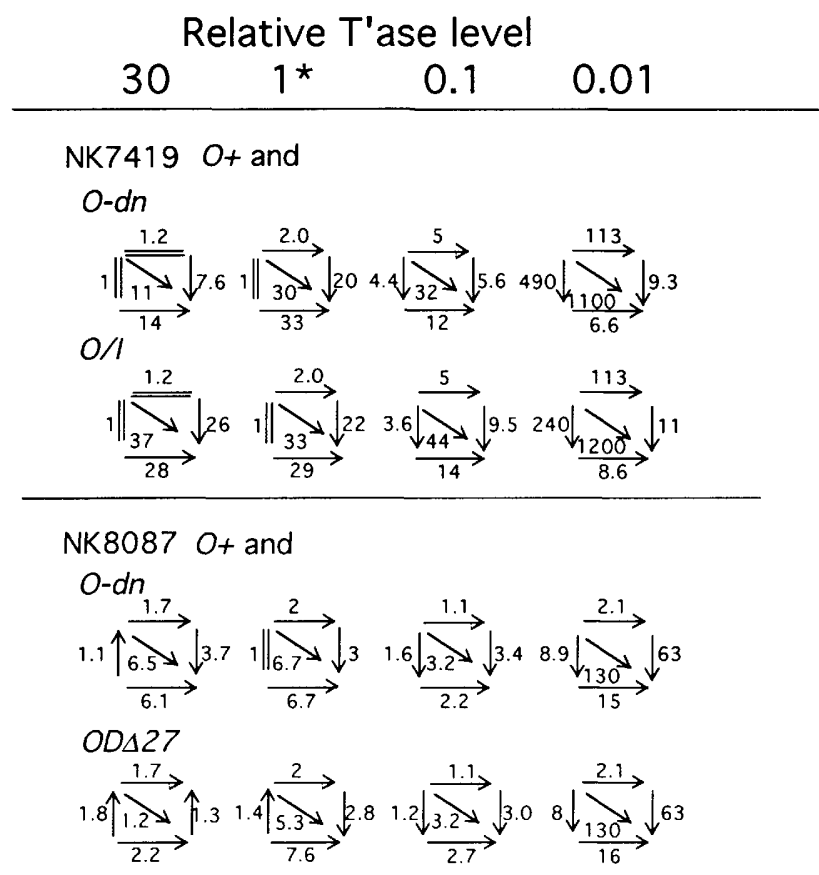

Figure 5. Intermolecular transposition from a multicopy plasmid at varying levels of transposase. Mini-Tn10-'kan ery $R$ plasmid constructs were assayed for transposition exactly as in Fig. 4 but at four different levels of transposase. Experiments are presented in two different strain backgrounds. Data for NK7419 and NK8084 (top) represents the average of four independent experiments. Data for NK8087 and NK8088 (bottom) is for one experiment. In this and other assays, these two strain backgrounds reproducibly give qualitatively similar but quantitatively distinguishable results. The standard transposase level was provided in trans from the Ptac-transposase plasmid pNK3358 as in previous figures. An $\sim 30$-fold higher level of transposase was achieved by inserting the Ptac-transposase fusion into each of the minitransposon plasmids, which were then assayed in the absence of any additional plasmid. A 10-fold reduction in transposase below the standard level was achieved by providing transposase from a weaker promoter; the corresponding Plac-transposase plasmid pNK3362 is otherwise isogenic to the Ptac-transposase plasmid. The host strains carry a single chromosomal copy of the lacI repressor gene, which provides a small degree of repression to the multicopy plasmid promoters. A reduction of $\sim 100$-fold in transposase level was achieved by insertion of a $l a c l^{\mathrm{Q}}$ gene into the Plac plasmid thus conferring essentially full repression. The relative effective levels of transposase provided in each of these situations have been determined previously (Way 1984) and are also apparent from absolute levels of transposition observed in the fully wild-type $\left(I \mathrm{HF}^{+} \mathrm{O}^{+}\right)$situation: $\left(\mathrm{NK} 7419: 2 \times 10^{-4}, 3 \times 10^{-5}, 7 \times 10^{-7}\right.$, $2 \times 10^{-9}$; NK8087: $3 \times 10^{-3}, 5 \times 10^{-4}, 2 \times 10-5,2 \times 10^{-6}$. The background level of kanamycin resistance observed in the absence of transposase was $<10^{-9}$.

observed in the NK8087 strain background at the standard transposase level and in both strain backgrounds at higher and lower transposase levels: In eight additional such experiments, increases varied from zero to fourfold (data not shown).

The chromosomal situation exhibits another interest-
Table 1. Transposition of minitransposons from a multicopy plasmid in $\mathrm{IHF}^{+}$and $\mathrm{IHF}^{-}$strains

\begin{tabular}{lccc}
\hline \multirow{2}{*}{$\begin{array}{l}\text { End } \\
\text { genotype }\end{array}$} & \multicolumn{3}{c}{ Host } \\
\cline { 2 - 4 } $\mathrm{IHF}^{+}$ & $\mathrm{IHF}^{-}$ & $\mathrm{IHF}^{-} / \mathrm{IHF}^{+}$ \\
\hline$O^{+}$ & $\equiv 1$ & 2.8 & 2.8 \\
$I 27^{\mathrm{a}}$ & 0.04 & 0.2 & 5 \\
$I 23^{\mathrm{a}}$ & 0.016 & 0.17 & 11 \\
$I 19^{\mathrm{a}}$ & 0.013 & 0.09 & 6.9 \\
\hline
\end{tabular}

Transposase was provided in cis by a Ptac-transposase fusion present on the same plasmid as the transposon ends. Transposition was assayed in NK7419 and NK8084 exactly as in Figs. 4 and 5. Plasmids were pNK2811 and pNK2817-2819. Data represent the average of three independent experiments, all of which gave very similar results. The absolute frequency of $\mathrm{Kan}^{\mathrm{R}}$ derivatives for the $O^{+}$case was $1.8 \times 10^{-4}$; the frequency of $\mathrm{Kan}^{\mathrm{R}}$ derivatives observed with an isogenic plasmid lacking transposase (pNK2824) was $<10^{-9}$.

anside-end activity is inhibited by GATC methylation in these Dam $^{+}$strains (Roberts et al. 1985)

ing and unique feature: Elimination of either IHF or the IHF-binding site individually confers a significant decrease in the frequency of transposition. But elimination of both determinants restores transposition to the original level. These effects are observed at all transposase levels and in both strain backgrounds (Fig. 6; data not shown|. These observations imply that for chromosomal transposition, the two unbalanced configurations are unfavorable for transposition (see Discussion).

\section{Tn10-promoted chromosome rearrangements are specifically activated by IHF-acting at the IHF-binding site}

Chromosome rearrangements exhibit yet a third pattern of responses to genetic perturbation of the IHF/end in-

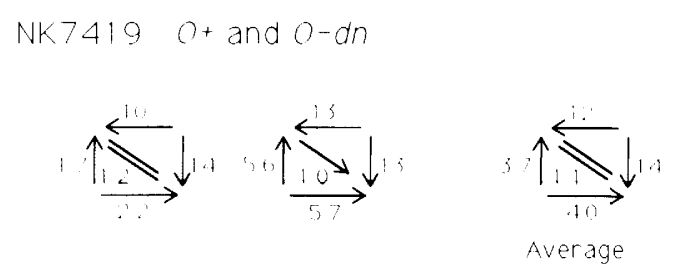

Figure 6. Intermolecular transposition from the chromosome. The same mini-Tn10-'kan ery $R$ constructs assayed on plasmids in Figs. 4-6 were assayed in the chromosome on single copy $\lambda$ prophages (Materials and methods). Transposase was provided at the standard level, in trans from the Ptac-transposase plasmid pNK3384. Results from two independent experiments involving NK7419 and NK8084, and their average, are shown. The absolute frequencies of kanamycin-resistant derivatives observed for the wild-type transposon in the $\mathrm{IHF}^{+}$strain were $2 \times 10^{-6}$ and $5 \times 10^{-6}$. The background frequency of kanamycinresistant derivatives obtained in the absence of transposase was $<10^{-9}$ 
teraction: They are specifically dependent on both IHF and its binding site.

In the absence of both relevant determinants, deletions and inversions both decrease in frequency. The extent of the decrease observed for the two types of rearrangements taken together ranges from 3-to 13-fold (Fig. 7). This decrease appears to be the result of the elimination of a simple positive effect of IHF binding to its specific site, irrespective of other factors. Elimination of either determinant singly confers nearly as great a decrease

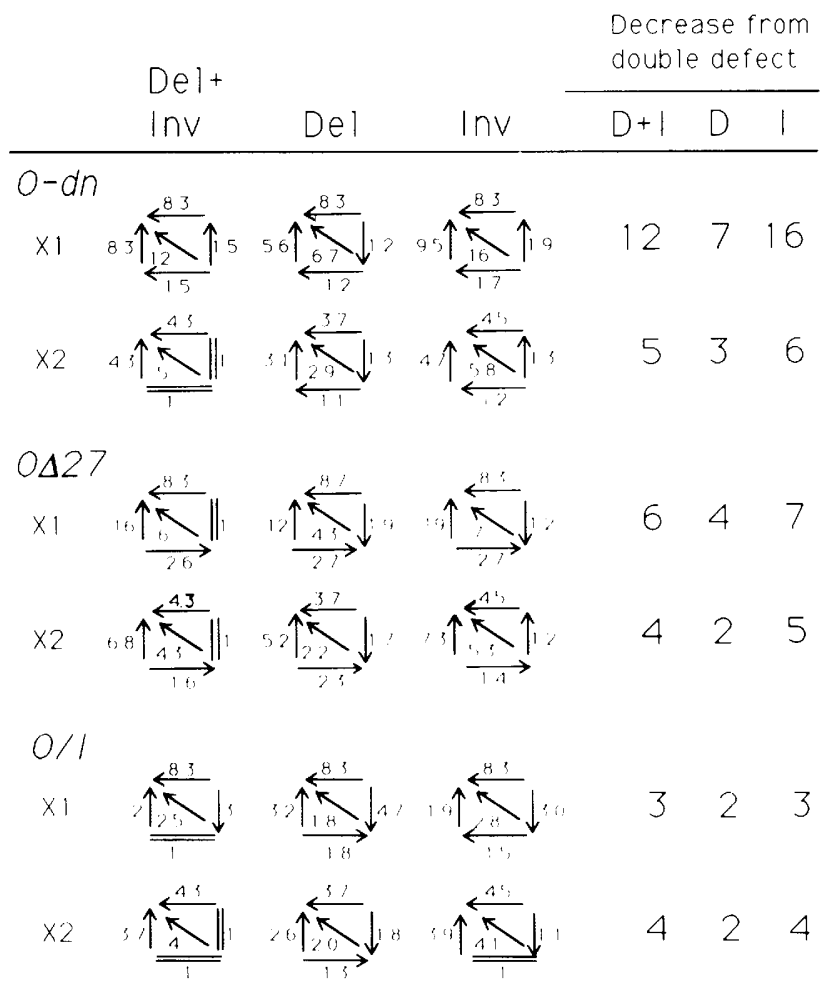

Figure 7. Assays of chromosome rearrangements. Rearrangement tester elements of the type described in Fig. 3 were made carrying $\mathrm{O}^{+}$and the three types of mutant ends shown, introduced into the bacterial chromosome on single-copy $\lambda$ prophages and analyzed in the presence of transposase at the standard level, provided in trans from the Ptac-transposase plasmid pNK3384 (Materials and methods; Fig. 3B). X1 and X2 are two independent experiments in the NK7419 strain background. In the two experiments, different strains were used that differ with respect to the orientation of the $\operatorname{sac} B$ gene within the tester construct: $\mathrm{X} 1$ and $\mathrm{X} 2$ involved constructs bearing the $s a c B$ gene in orientation I and orientation II, respectively (Materials and methods). Similar results have been obtained in the NK8087 strain background (data not shown). The absolute frequencies of Suc $^{\mathrm{R}} \mathrm{Tet}^{\mathrm{R}}$ derivatives observed for the construct having $\mathrm{O}^{+}$ ends in the $\mathrm{IHF}^{+}$strain for the two experiments were $0.5 \times 10^{-3}$ (X1) and $1.3 \times 10^{-3}(\mathrm{X} 2)$; the proportions of inversions and deletions in these two cases were $78 \%$ and $22 \%(\mathrm{X} 1)$ and $84 \%$ and $16 \%(\mathrm{X} 2)$. The background levels of $\mathrm{Suc}^{\mathrm{R}} \mathrm{Tet}^{\mathrm{R}}$ derivatives obtained for this construct in the presence of a Tase-plasmid, pNK3386, were $2 \times 10^{-6}(\mathrm{X} 1)$ and $7 \times 10^{-7}(\mathrm{X} 2)$, two or more orders of magnitude lower than the lowest levels observed in the presence of transposase. as elimination of both determinants (compare the leftmost horizontal and vertical comparisons with the diagonal comparison).

An additional feature of the data hints, however, at the presence of additional complexities: The effect of eliminating determinants is reproducibly twofold greater for inversions than for deletions (Fig. 7; summary on right). Random collision between synapsed transposon ends and target DNA is expected to yield deletions and inversions with equal frequency; in this pathway, the two events are mechanistically indistinguishable, differing only in the orientation with which the target DNA happens to interact with the ends. Correspondingly, any genetic alteration that affects this collision pathway is expected to affect both types of events to the same extent. The observation that inversions are affected to a greater extent than deletions is consistent with the existence of an IHF-dependent pathway that leads only to inversions (a channeling pathway; see Discussion).

\section{Constructs analogous to Tn 10 and IS10 respond like $\mathrm{O}^{+}$constructs to elimination of IHF}

The combinations of IS10 ends that occur in nature include the pair of slightly different outside ends found in wild-type $\operatorname{Tn} 10(L x O)$ and the combination of inside and outside ends found in IS10-Right $(I x O)$ as well as the combination of two inside ends (which are identical for IS10-Right and IS10-Left; Halling et al. 1982). Thus, minitransposon constructs corresponding to Tn10 and IS10 have been analyzed. Both constructs behave similarly to the model outside-end constructs described above. With respect to intermolecular transposition from a plasmid, elimination of IHF function confers the same $\sim 10$-fold increase in on the wild-type Tn10 analog $(L x O)$ as on the $O^{+}(O x O)$ element analyzed above. With respect to chromosomal rearrangements, $\operatorname{Tn} 10(\mathrm{LxO})$ and IS10-Right $(I x O)$ constructs exhibit the same $\sim 5$ - to 10 -fold reduction as the $(\mathrm{OxO})$ element.

Also, with respect to chromosome rearrangements, the Tn10 inside-end analog $(I x I)$ exhibits a small (twofold) decrease in an $\mathrm{IHF}^{-}$strain as compared with $\mathrm{IHF}^{+}$ (data not shown). The existence of a small effect on ends that do not contain an IHF-binding site supports the view that IHF can act nonspecifically to promote rearrangements.

Transposase expression in the chromosome is not affected by the absence of IHF

In an earlier analysis, Roberts (1986) examined the effects of an $\mathrm{IHF}^{-}$mutation on the frequency of rearrangements promoted by an IS10-based transposon composed of a gal operon carrying a galE $E^{-}$point mutation that is flanked by IS10-Right and IS10-Left in direct repeat. This transposon, nadA::TnGal, arose from a nadA::Tn10 insertion via a $\operatorname{Tn} 10$-promoted inversion event, and it has exactly the structure of Tn- $d c b a$ of Figure 2 with $d c b a$ corresponding to gal operon sequences/Raleigh and 
Kleckner 1984). The inside ends of TnGal involved in promoting chromosome rearrangements are, genetically, the inside end of IS10-Left and the outside end of IS10Right.

For nadA::TnGal, elimination of IHF results in a 5- to 10 -fold reduction in the frequency of rearrangements (Table 2), precisely the same effect as that reported above for mini-Tn10 $(O x L)$ despite differences in the strain background, the transposon construct, and the decade in which the experiments were performed. More importantly, exactly the same reduction is observed irrespective of whether transposase was provided from the transposon's own $p I N$ promoter or at a much higher level from a Ptac-transposase fusion present on a multicopy plasmid. Taken together with current data, these observations imply that for IS10 elements in the chromosome, the presence or absence of IHF has little or no effect on the level of transposase gene expression.

More direct evidence to this effect is provided by analysis of transposase-lacZ translational fusions in $\mathrm{IHF}^{+}$ and $\mathrm{IHF}^{-}$hosts (D.E. Roberts, pers. comm.). When such a fusion is present on a multicopy (pUC) plasmid, the level of expression in an $\mathrm{IHF}^{-}$strain is sixfold higher than in $\mathrm{IHF}^{+}$; in contrast, when the fusion is present in the chromosome on a single-copy $\lambda$ prophage, elimination of IHF causes an increase of approximately twofold in expression. And severalfold differences between plasmid and chromosome with respect to the effects of IHF elmination are observed when the fusion construct contains a mutation that improves the IHF-binding site (IHF-up; Huisman et al. 1989) or an I/O hybrid terminus with or without the IHF-up mutation.

\section{Discussion}

We interpret the results presented above to mean that IHF and its binding site participate in two different reg-

Table 2. Frequency of $\mathrm{Gal}^{R}$ chromosome rearrangements promoted $\operatorname{Tn}(\mathrm{Gal})\left(\times 10^{5}\right)$

\begin{tabular}{llrcc}
\hline & \multirow{2}{*}{$\begin{array}{l}\text { Tase } \\
\text { Experiment }\end{array}$} & \multicolumn{3}{c}{ Strains $^{\mathrm{b}}$} \\
\cline { 2 - 5 } & IHF $^{+}$ & IHF & IHF $^{+} / \mathrm{IHF}$ \\
\hline 1 & none & 46 & 6.6 & 7.0 \\
2 & none & 42 & 7.2 & 5.8 \\
& Ptac-taseNcos & 37 & 12 & 3.1 \\
3. & Ptac-tase & 1450 & 250 & 5.8 \\
& none & 50 & 5.7 & 8.8 \\
& Ptac-taseNcos & 67 & 11 & 6.1 \\
& Ptac-tase & 1450 & 360 & 4.0
\end{tabular}

The gal genes of Tn Gal carry a galE mutation that renders cells sensitive to galactose. Cultures were grown in LB from single colonies to saturation, diluted, and plated in parallel on minimal media containing either glucose /to measure total colony forming units) or glucose plus galactose (to measure $\mathrm{Gal}^{\mathrm{R}}$ colony forming units; Raleigh and Kleckner 1984).

a Ptac-tase is pNK474; Ptac-taseNcoD is pNK534, a deletion derivative of pNK474 that lacks the central portion of the transposase coding region.

bN7381 (=W3110 TnGal) and an isogenic himA::TN10 hip:: cat derivative. ulatory processes, one negative and one positive, which are directed at two different aspects of Tn10/IS10 biology. Thus, as in many other biological systems, appropriate regulation is achieved by the combined effects of opposing positive and negative tendencies.

\section{Negative regulation specifically inhibits plasmid transposition}

Tn10 transposition off of a multicopy plasmid is subject to negative regulation at two levels, neither of which significantly affects Tn10 transposition off of the chromosome.

First, as shown here, IHF and its binding site act directly on the reaction process to inhibit intermolecular transposition: For a mini-Tn10 element on a multicopy plasmid donor replicon, elimination of IHF and its cognate binding site at the outside end of IS10 results in a 10- to 30-fold increase in intermolecular transposition. These same genetic perturbations have relatively little effect on transposition from the chromosome.

Second, previous work has shown that, as a separate effect, IHF inhibits transposase expression for an IS10 element on multicopy plasmids (Huisman et al. 1989; Sussman 1992; Ditto et al. 1994). The data presented here suggest that no such inhibition occurs for an IS10 element located in the bacterial chromosome.

Thus, as for several other IS10 regulatory processes, host factor-mediated regulation is directed at both transposase expression and the reaction process per se.

Previous work has identified several general regulatory features that cause transposition frequencies to decrease as the total number of transposon copies per cell increases. Among these, the most important is probably concentration-dependent inhibition of transposase gene expression by an IS10-encoded antisense RNA; in combination with preferential cis action of transposase, this feature is sufficient to keep the total number of transposition events per genome per generation constant over a wide range of transposon copy numbers (Simons and Kleckner 1983, 1988). Despite these controls, however, transposition of an IS element into a multicopy plasmid replicon poses an immediate risk that a cell lineage will undergo an explosion of transposition events. The host factor-mediated regulation described here presumably provides specific additional barriers against this particularly dangerous possibility.

The fact that negative regulation is exaggerated at very low transposase levels should also mean that sporadic, unscheduled expression of a few transposase molecules from a plasmid-borne IS10 element will essentially never lead to the occurrence of transposition.

\section{Mechanism of negative regulation}

IHF-mediated negative regulation in vivo appears to correspond to an in vitro phenomenon known as channeling. The transposition process as observed on a supercoiled plasmid minitransposon $\mathrm{OxO}$ substrate does not 
require accessory host factors $(R$. Chalmers and $N$. Kleckner, unpubl.). Inclusion of physiologically sensible concentrations of either IHF or HU cause a change in the array of products observed: Transposon excision occurs normally, but strand transfer products are predominantly or exclusively unknotted intratransposon inversion circles (Benjamin and Kleckner 1989; H. Benjamin, R. Chalmers and N. Kleckner, unpubl.). This bias implies that ends are finding target DNA in a topologically constrained way. Intermolecular transpositions and intratransposon events that arise by random collision of ends with target DNA (e.g., deletions) are suppressed.

The correspondence between the in vivo and in vitro phenomena is supported by previous Southern blotting analysis of products generated in vivo from plasmidborne mini-Tn10 elements in which the only intratransposon products observed were inversion circles; deletion circles, which arise by a collision mechanism, were absent (Benjamin and Kleckner 1989). This bias for inversions implies that such substrates are subject to strong channeling effects in vivo.

Furthermore, the $\mathrm{O}^{+}$plasmid substrate described above responds in the expected way to elimination of IHF or the IHF-binding site. The $O d n$ and $O / I$ mutations both result in a 10-fold decrease in the level of inversion circles as determined by Southern blot hybridization of DNA extracted from saturated cultures, whereas in the same cultures, intermolecular transposition was increased in the case of $\mathrm{IHF}^{-}$as compared with $\mathrm{IHF}^{+}$as shown above (L. Signon, unpubl.). Fourth, all of the complexities of negative regulation observed in vivo either correspond to or are easily rationalized by features of the channeling reaction obscrved in vitro (A. Guhathakurta, R. Chalmers, H. Benjamin, and N. Kleckner, in prep.). Specifically, (1) negative regulation by IHF in vivo occurs on both outside and inside ends (above); the same is true in vitro. (2) For outside IS10 ends that retain the IHFbinding site, elimination of IHF function alone is not sufficient to fully relieve the negative regulation. This observation can be explained if $\mathrm{HU}$ can substitute for IHF for negative regulation. And both IHF and $\mathrm{HU}$ are effective for channeling on such ends in vitro. (3) Conversely, for outside IS10 ends in an $\mathrm{IHF}^{+}$strain background, elimination of the IHF-binding site alone is not sufficient to fully relieve the negative regulation. This observation implies that IHF is capable of mediating negative regulation in the absence of an IHF-binding site. And in vitro, IHF can promote channeling on inside ends even though they lack a specific IHF-binding site. (4) Elimination of both IHF and the IHF-binding site fully relieves the negative regulation despite the presence of $\mathrm{HU}$. This observation suggests that $\mathrm{HU}$ is capable of mediating channeling only if a wild-type IHF-binding site is present. This is not unlikely because the IHF-binding site not only provides sequence-specific contacts needed for specific IHF binding but also an intrinsic tendency to bend, which should promote binding of $\mathrm{HU}$ as well as of IHF. Furthermore, a mutation that increases the affinity of IHF for this site also increases the effectiveness of HU action at this site (D. Morisato and N. Kleckner, un- publ.); thus, it is not unreasonable to suppose that mutations that decrease the affinity of IHF for this site should decrease the effectiveness of HU.

\section{Positive regulation of chromosome rearrangements}

Positive regulation by IHF and its binding site is especially prominent for deletions and inversions promoted by a chromosomal element: The same genetic perturbations that lead to an increase in transposition off of a plasmid lead instead to a 5- to 10 -fold decrease in the frequency of chromosome rearrangements.

The mechanism of positive regulation is distinct from that of negative regulation. Activation of chromosome rearrangements appears to involve the specific interaction of IHF at its binding site, as elimination of either determinant singly confers essentially the same effect as elimination of both determinants. The activated rearrangements include both inversions and deletions. Thus, activation occurs for events promoted by the same collision pathway that yields intermolecular transpositions, as deletions and inversions are both generated by a process in which synapsis of transposon ends followed by random collision with target DNA (Benjamin and Kleckner 1992; R. Chalmers and N. Kleckner, in prep.).

Interaction of IHF with its binding site should also promote transposition of long composite transposons

The existence of a difference in the genetic dependencies for chromosome rearrangements and for transposition is particularly interesting because both types of events occur by a random collision pathway. Thus, the only apparent difference between the two types of events is the length of the segment between the synapsed ends, which is a few kilobases in the case of transposition but is the size of the Escherichia coli genome in the case of rearrangements (Fig. 2). These considerations suggest that IHF should also be required for transposition when the transposing segment is very long.

Mechanistically, IHF might be specifically required for formation or stabilization of the $\operatorname{Tn} 10$ synaptic complex when the segment between the two transposon ends is very long. It would not be surprising if a longer transposon segment imposed special physical stresses or constraints during or after synaptic complex formation.

It is already known that the transposition reaction can be sensitive to transposon length. The frequency of transposition, even for elements the size of Tn10 or less, in a wild-type strain, decreases exponentially with increasing transposon length, $\sim 40 \%$ per kilobase (Morisato et al. 1983). This transposition length dependence is also observed with IS1-based composite transposons (Chandler et al. 1982). In the case of Tn10, further analysis suggests that length dependence arises prior to transposon excision (Flick 1991) It would be interesting to determine whether this normal length dependence is increased in an $\mathrm{IHF}^{-}$strain and/or with outside ends that lack an IHF-binding site. 


\section{Transposition from the chromosome}

Chromosomal transposition events are subject to negative regulation, presumably at least in part via channeling: Transposition from the chromosome increases about twofold in the absence of both IHF and a functional binding site. Transposition from the chromosome is also subject to positive regulation: If either IHF or the IHF-binding site is eliminated singly, transposition from the chromosome is markedly decreased.

The level of transposition observed in these singly defective cases is very low. There are two possible explanations for this result. First, the chromosome may be the subject of both strong positive and strong negative regulation, which are essentially independent of one another. Elimination of positive regulation in the singly defective cases reveals the existence of the strong negative regulation; and when both are eliminated, the situation is not very different from when both are present. Second, it is possible that in the wild-type situation, where positive regulation is present, negative regulation is negligible, and that strong negative regulation occurs only in the absence of positive activation.

\section{Some fraction of chromosome rearrangements may be} channeled into an inversion-only pathway

Elimination of both IHF and a functional IHF-binding site causes a twofold greater decrease in chromosomal inversions than in chromosomal deletions. In the fully wild-type situation, the ratio of inversions to deletions is $\sim 4: 1 \quad(3: 1$ and $5: 1$ in $X 1$ and $X 3$, respectively); in the absence of both IHF and its binding site, the ratio of inversions to deletions is $\sim 2: 1$ (average of all data in Fig. 7 ; range is $1.3: 1$ to $2.5: 1)$. Because inversions and deletions that occur by the collision pathway should be mechanistically indistinguishable, differing only in the orientation with which the target DNA and the transposon ends happen to interact, the differential behavior of the two types of rearrangements suggests the existence of some other variable.

One simple possibility would be that in a fully wildtype situation, some inversion events arise by the inversion-specific channeling pathway. The quantitative effects of eliminating both IHF and its binding site are consistent with the possibility that about one-third of all chromosome rearrangements are normally channeled. In the fully wild-type case, $20 \%$ of rearrangements are deletions and $80 \%$ are inversions /average of $\mathrm{X} 1$ and $\mathrm{X} 2$; legend to Fig. 7). If all deletions arise by collision and if the ratio of inversions to deletions via the collision pathway (given by the ratio in the absence of IHF and its binding site) is $2: 1$, then in the wild-type case, $25 \%$ of events are collision deletions, $50 \%$ are collision inversions, and the remaining $25 \%$ of events are inversions that arrive by channeling. This estimate predicts that the ratio of inversions to deletions in the presence and in the absence of channeling should be 2.35:1 (not shown); the observed ratio is $2.3: 1$

If this scenario were exactly true, and if the rules for channeling observed on plasmids apply directly to chromosomal events as well, single mutant defects (in IHF or the IHF site) should differentially affect deletions (which arise only by collision) as compared with inversions (which arise by both collision and channeling). This simple expectation is not borne out.

\section{IHF should specifically promote the formation of new composite transposons}

Taken together, the observations presented here suggest that the interaction of IHF with its binding site should play an important role in both the formation and the transposition of new composite transposons. A new transposon can arise in either of two ways, both of which should be stimulated by IHF.

In one process, an individual IS10 element may arise at one or more new locations such that a gene of interest is flanked by two new or one new and one old element. In the most general case, the resulting newly formed transposon encoding the gene of interest will be very long, because both the pre-existing element and any new IS10 insertions will be randomly disposed with respect to that gene (e.g., Wolf 1980). Thus, if IHF generally promotes transposition of long composite elements, it will be particularly important for subsequent transposition of newly formed composite elements. The IHF dependence will eventually be lost as the newly formed transposon evolves. Because transposition is more efficient for shorter elements, newly formed elements will tend to become progressively shorter as they undergo successive rounds of transposition.

In a second process, an existing pair of IS10 elements undergoes a chromosome rearrangement that places new sequences between the elements, as in Tn10-promoted inversions (Fig. 2). IHF should promote the formation of such rearrangements both by acting as a positive factor for inversions that arise by collision and, perhaps more especially, by specifically channeling some collision events into the topologically constrained pathway that yields only inversions.

\section{Why is a multicopy plasmid different from} the chromosome

The only feature of IHF-mediated regulation for which a possible explanation is not immediately available is that plasmid substrates are differentially sensitive to negative regulation of both transposase expression and transposition as compared with chromosomal substrates. An obvious possibility is that the steady-state level of negative supercoiling in plasmids might be higher than for the chromosome, because supercoiling would presumably promote a topologically constrained reaction pathway. In vivo estimates of effective supercoiling levels suggest, however, that plasmid and chromosomal substrates are similar in this regard (Sinden et al. 1980; Bliska and Cozzarelli 1987). Local supercoiling effects (Liu and Wang 1987) also seem unlikely because different types of con- 
structs were used in current and previous (Benjamin and Kleckner 1989) studies.

A second possibility would be that plasmids and chromosomes have different arrays of chromatin structure proteins, perhaps as a consequence of differences in plasmid and chromosomal replication. A third possibility would be that the length of the nontransposon sequences in the substrate molecule affects the nature of the reaction subsequent to synaptic complex formation (e.g., Wang and Harshey 1994).

\section{Materials and methods}

\section{Bacterial strains}

Strains used for assaying transposition off of plasmids NK7419, trp his arg met supE44 tonA tsx mtl2 xyl7 lac $\Delta r 1$; NK8084, NK7419 himA::Tn10482 43hip::cat; NK8087, galK2 thyA strR; NK8088, NK8087 himA::Tn10482 $\Delta 3$ hip::cat; himA:: Tn10482 and $\Delta 3$ hip::cat, as described by Miller (1984) and Flamm and Weisberg (1985), respectively; NK7814, W3110 strR lac $\Delta r 1 ; \mathrm{NK} 7817$, NK7814 himA::Tn10482 hip 11; DRC325, recA56 lacZU169 galK2 rpsL200; DRC324, DRC325 himA4SmaI $\triangle 3$ hip::cat.

Strains used for experiments involving transposons carried as $\lambda$ prophages For all experiments that assay chromosome rearrangements and for assays of chromosomal transposition in which transposase was provided in trans, lysogens of the desired $\lambda$ phages were obtained in NK7419 and/or NK8087 by infection of each phage and selection for a phage-encoded erythromycin resistance marker (see below). After purification, single lysogens were identified by ter testing (Mousset and Thomas 1969); then an isogenic IHF (hip::cat) version of each desired single lysogen was constructed by $\mathrm{Pl}$ transduction. The $\operatorname{him} A$ :: Tn10 182 marker was not introduced because it was incompatible with the selection for chromosome rearrangements. All available evidence suggests that elimination of a single IHF subunit is sufficient to eliminate all IHF activity (Werner et al. 1994; Zulianello et al. 1994).

In experiments involving multicopy plasmids, plasmids were introduced in monomeric form into the desired strain by transformation. Fresh transformants were generated for each experiment as the first steps in the procedure (see below). Transposon-bearing plasmids and transposase-bearing plasmids were introduced and maintained by selection for erythromycin and ampicillin resistance, respectively. For experiments involving both types of plasmids, the transposase plasmid was introduced first by selection for ampicillin resistance and the transposonbearing plasmid was introduced subsequently by selection for erythromycin resistance in the presence of ampicillin.

\section{Plasmids}

Multicopy plasmids carrying mini-Tn $100^{\prime}$ kan ery $R$ transposons and a Ptac-transposase gene are derivatives of pBR333 (Foster et al. 1981), a medium copy derivative of pBR322 that retains ampR: $\quad$ pNK2811(O $\left.{ }^{+}\right), \quad$ pNK2823(O-dn), pNK3348(O427), pNK3350(O 423$)$, pNK2812(O/I), pNK2820 (I/O), pNK2816 (I/ O-dn), pNK2819 (I27), pNK2818 (I23), and pNK2817 (I19). These plasmids were constructed in a single step by ligation of four pieces of DNA: (1) An EcoRI-HindIII backbone fragment from precursor plasmid pLO88, which carries the origin and amp gene of pBR333, the Ptac-transposase gene and a set of transcription terminators derived from pNK1391; the latter segments are embedded within a segment encoding the hisOPGD region of Salmonella typhimurium. (2) An EcoRI-Sall fragment carrying the desired type of IS10 end; (3) a XhoI-BamHI fragment from pNK3505 carrying the 'kan and eryR genes; and (4) a BclI-HindIII fragment carrying the desired type of IS10 end. The ends on fragments 2 and 4 may be the same or different. In these constructs, the transposase gene was transcribed from a Ptac promoter located $1.5 \mathrm{~kb}$ from and directing transcription away from the nearest transposon end. pNK3505 was constructed as follows. A BamHI-Sall fragment containing the 'kan gene from pKM109-9 (Reiss et al. 1984) was cloned into pGCl (Myers et al. 1985) to yield pNK3504. Plasmid pGI4010 carrying the erythromycin resistance gene (Josson et al. 1989) was digested with SalI and SacI, its overhanging ends filled in, and the resulting fragment was cloned into a filled-in Sall site in pNK3504 to yield pNK3505.

Isogenic transposase-defective derivatives of the above plasmids were constructed in analogous four-piece constructions differing from the above only in that the transposase gene on fragment 1 had been inactivated by digestion with AccI, filling in the overhanging ends, and inserting a Sall 8-mer linker. The corresponding Tase ${ }^{-}$plasmids are pNK2824 $\left(\mathrm{O}^{+}\right)$, pNK3344 (O$d n), \mathrm{pNK} 3346(O / I), \mathrm{pNK} 3354(O \Delta 23)$, and pN3352 (O $\Delta 27)$.

Plasmids that encode only a transposase gene and no transposon are as follows: The Ptac-transposase plasmid pNK3358 was obtained by the cloning of the BamHI-HindIII fragment of pDH10 (Haniford et al. 1990) into pACYCl77. The Plac-transposase plasmid pNK3362 was obtained by cloning the EcoRI fragment of pNK997 (Shen et al. 1987) into pACYC177. A derivative of pNK3362 containing a lacI $\mathrm{P}^{\mathrm{Q}}$ gene, pNK3364, was obtained by inserting an EcoRI fragment of pNK627 (obtained from J. Wang, Harvard University, Cambridge, MA) at the EcoRI site of pNK3362.

Assays of chromosome rearrangements involved plasmids that carried both a transposase gene and a $P_{\mathrm{R}}-t e t R$ fusion (Roberts et al. 1980). An Aval (filled in)-HindIII fragment of pTR264 carrying the $P_{\mathrm{R}}-t e t R$ fusion was cloned into each of the transposase plasmids described above following digestion with HindIII and SmaI. pNK3358 (Ptac) yielded pNK3384; pN3362 (Plac) yielded pNK3368; pNK3364 yielded pNK3370. pNK3386 is isogenic to pNK3384 except that it lacks a functional transposase gene; it was constructed by the replacement of the BamI-HindIII fragment of pNK3358 with the corresponding fragment carrying the Ncols allele from pDH12 (Haniford et al. 1989) after insertion of the $P_{\mathrm{R}}-t e t R$ fusion fragment. pNK3390 and pNK3392 are isogenic to pNK3368 except that they lack a functional transposase gene; these plasmids were constructed by the replacement of the EcoRI-HindIII fragments of pNK3362 and pNK3370, respectively, with a fragment carrying a Plactransposase NcoI $\Delta$ allele from pNK536.

\section{Phages}

For assays of transposition from the chromosome, transposon constructs were transfered by recombination in vivo from the plasmids described above to a lysogenization-proficient $\lambda$ phage, $\lambda 1371=\lambda$ hisGD imm434 ind $^{-}$.

For assays of chromosome rearrangements, the assay tester construct was first constructed on a plasmid and then transferred to $\lambda 1371$ by recombination in vivo. The tester construct for any given type of ends was obtained in three steps. (1) Four fragments were ligated together: A SalI-PvuII backbone derived from pNK2242 (a derivative of pBR333), an EcoRI-PvuII fragment from the appropriate plasmid of the pNK2811 series (see above) containing one end plus the eryR gene, a HindIII-SalI 
fragment also from the appropriate plasmid of the pNK2811 series carrying one end plus the 'kan gene, and a EcoRI-HindIII ended fragment carrying a PlacUV5-CI fusion gene; the latter fragment was derived by partial digestion of a plasmid similar to pEA306 (Amann et al. 1983). (2) A NotI linker was then inserted at the EcoRI site located between the PlacUV5-cI fragment and the adjacent ery $R$ terminus segment, and (3) a NotI fragment carrying the $s a c B$ gene from pBIp (Slater and Maurer 1993) and subcloned into pBluescript. For each construct, plasmids carrying the $s a c B$ fragment in both possible orientations were kept. In orientation $\mathrm{I}$, the $\operatorname{sac} B$ gene is transcribed in the same direction as the PlacUV5-CI gene; orientation II is the oppposite.

For orientation I, plasmids are pNK3409 $1 \mathrm{O}^{+}, \mathrm{pNK} 3411$ $(O x L)$, pNK3413 (O-dn), pNK3414 (O/I), pNK3416 (OxI), pNK3419 (I27), and pNK3418 (O427). For orientation II, the plasmids are pNK3423 $\left(O^{+}\right)$, pNK3425 (OxL), pNK3427 (O-dn), pNK3429 (O/I), pNK3430 (OxI), pNK3432 (I27), and pNK3433 (O $\Delta 27)$. For orientation I, the corresponding phages are $\lambda 1395$ $\left(O^{+}\right), \lambda 1397(O x L), \lambda 1399$ (O-dn), $\lambda 1401$ (O/I), $\lambda 1405$ (OA27), and $\lambda 1407$ (I27). For orientation II, the corresponding phages are $\lambda 1409\left(O^{+}\right), \lambda 1411(O x L), \lambda 1413(O-d n), \lambda 1415(O / I), \lambda 1417$ $(O x I), \lambda 1419$ (I27), and $\lambda 1421$ (OS27).

\section{Transposition and chromosome rearrangement assays}

Transposition by 'kan elements was assayed as follows. For each strain to be tested, three or five single colonies representing primary transformants containing the plasmids of interest were picked from an LB plate containing erythromycin and ampicillin; each colony was used to inoculate $1 \mathrm{ml}$ of LB broth containing ampicillin. Cultures were grown 12 to $14 \mathrm{hr}$ with aeration and then titered on LB ampicillin plates in the presence and absence of kanamycin. Results presented for each strain in any single experiment are the average of the three to five independent clones assayed.

Chromosome rearrangements were assayed analogously except that the final cultures were titered on LB ampicillin plates in the presence or absence of tetracycline (for lysogens in NK7419 and their isogenic IHF derivatives) or on LB ampicillin plates in the presence or absence of sucrose (for lysogens in NK8087 and their isogenic IHF derivatives). The desired Tet ${ }^{R}$ $\mathrm{Suc}^{\mathrm{R}}$ derivatives were identified in the former case by replica plating of tetracycline-resistant colonies onto sucrose-containing plates and in the latter case by replica plating of sucroseresistant colonies onto tetracycline-containing plates. The protocol was varied for NK8087 because this strain exhibited a high background of irrelevant tetracycline resistance. The frequency of $\mathrm{Tet}^{\mathrm{R}} \mathrm{Suc}^{\mathrm{S}}$ and $\mathrm{Suc}^{\mathrm{R}} \mathrm{Tet}^{\mathrm{S}}$ colonies was negligible $(<6 \%)$ in all experiments reported here except in strains lacking transposase in which case only background events occur. In all cases, only Suc $^{\mathrm{R}} \mathrm{Tet}^{\mathrm{R}}$ colonies were considered in the frequencies reported.

The presence of the ery $R$ marker was assayed by replica plating all colonies onto LB plates containing erythromycin. This approach is subject to two uncertainties: Very short adjacent deletions that do not extend into the ery $R$ marker would have been scored as inversions and, conversely, inversions to a target site within the ery $R$ marker would have been scored as deletions. Previous analysis of Tn 10 -promoted rearrangements suggests that such events should constitute a small proportion of the total, however (Kleckner et al. 1975; Raleigh and Kleckner 1984; Shen et al. 1987). The presence of the 'kan marker was scored by colony hybridization (Bender et al. 1991).

\section{Methods and media}

All experiments were carried out using standard microbiological procedures (Miller 1972; previous publications from the
Kleckner laboratory|. Antibiotics were used at the following concentrations: $100 \mathrm{mg} / \mathrm{ml}$ of ampicillin; $50 \mathrm{mg} / \mathrm{ml}$ of kanamycin; $200 \mathrm{mg} / \mathrm{ml}$ of erythromycin; $10 \mathrm{mg} / \mathrm{ml}$ of tetracycline; and $5 \%$ sucrose.

\section{Acknowledgments}

L.S. and the research described in this paper were supported by a National Institutes of Health grant to N.K. (RO1-GM 25326). A National Science Foundation (INT-8715027-US-France Cooperative Science Program) to N.K. helped defray travel costs. We thank Sean Burgess, Ronald Chalmers, Janice Sakai, and Howard Nash for thoughtful reading of this manuscript, R.C. and J.S. for permitting citation of results prior to publication, and Jim Henle for manuscript preparation. L.S. gratefully acknowledges comments and support from Ariane Toussaint and Michael Chandler. This paper is dedicated to the memory of Olivier Huisman.

The publication costs of this article were defrayed in part by payment of page charges. This article must therefore be hereby marked "advertisement" in accordance with 18 USC section 1734 solely to indicate this fact.

\section{References}

Amann, E., Brosius, J. and M. Ptashne. 1983. Vectors bearing a hybrid trp-lac promoter useful for regulated expression of cloned genes in Escherichia coli. Gene 25: 167-178.

Baker, T.A. and K. Mizuuchi. 1992. DNA-promoted assembly of the active tetrameter of the Mu transposase. Genes \& Dev. 6: 2221-2232.

Bender, J. and N. Kleckner. 1986. Genetic evidence that Tn10 transposes by a nonreplicative mechanism. Cell 45: 801815 .

Bender, J., I. Kuo, and N. Kleckner. 1991. Genetic evidence against intramolecular rejoining of the donor DNA molecular following IS10 transposition. Genetics 128: 687-694.

Benjamin, H. and N. Kleckner. 1989. Intramolecular transposition by Tn10. Cell 59: 373-383.

1992. Tn 10 transposase excises Tn 10 from flanking donor DNA by flush double strand cleavages at the transposon termini. Proc. Natl. Acad. Sci. 89: 4648-4652.

Bisercic, M. and H. Ochman. 1993. The ancestry of insertion sequences common to Escherichia coli and Salmonella typhimurium. I. Bacteriol. 175: 7863-7868.

Bliska, J.B. and N.R. Cozzarelli. 1987. Use of site-specific recombination as a probe of DNA structure and metabolism in vivo. J. Mol. Biol, 194: 205-218.

Bonnefoy E., M. Takahashi, and J.R. Yaniv. 1994. DNA-binding parameters of the HU protein of Escherichia coli to cruciform DNA. J. Mol. Biol. 242: 116-129.

Broyles, S.S. and D.E. Pettijohn. 1986. Interaction of the Escherichia coli HU protein with DNA: Evidence for formation of nucleosome-like structures with altered DNA helical pitch. I. Mol. Biol. 187: 47-60.

Chalmers, R.M. and N. Kleckner. 1994. Tn10/IS10 transposase purification, activation and in vitro reaction. I. Biol. Chem. 269: $8029-8035$.

Chandler, M., M. Clerget, and D.J. Galas. 1982. The transposition frequency of IS1-flanked transposons is a function of their size. I. Mol. Biol. 154: 229-243.

Ditto, M.D., D. Roberts, and R.A. Weisberg. 1994. Growth phase variation of integration host factor level in Escherichia coli. I. Bacteriol. 176: 3738-3748.

Flamm, E. and R.A. Weisberg. 1985. Primary structure of the hip 
gene of $E$. coli and of its product, the $\beta$ subunit of integration host factor. J. Mol. Biol. 197: 117-128.

Flick, K. 1991. "The relationship between element length and transposition frequency of Tn10 in vivo and in vitro." Undergraduate honors thesis, Committee on Degrees in Biochemical Sciences, Harvard College, Cambridge, MA.

Foster, T., M.A. Davis, K. Takeshita, D.E. Roberts, and N. Kleckner. 1981. Genetic organization of transposon Tn10. Cell 23: 201-213.

Gay, P., D. LeCoq, M. Steinmetz, T. Berkelman, and C.I. Kado. 1985. Positive selection procedure for entrapment of insertion sequence elements in Gram-negative bacteria. I. Bacteriol. 164: 918-921.

Halling, S.M. and N. Kleckner. 1982. A symmetrical six-basepair target site sequence determines Tn 10 insertion specificity. Cell 28: 155-163.

Halling, S.M., R.W. Simons, J.C. Way, R.B. Walsh, and N. Kleckner. 1982. DNA sequence organization of Tn10's IS10-right and comparison with IS10-left. Proc. Natl. Acad. Sci. 79: 2608-2612.

Haniford, D.B., A.R. Chelouche, and N. Kleckner. 1989. A specific class of IS10 transposase mutants are blocked for target site interactions and promote formation of an excised transposon fragment. Cell 59: 385-394.

Haniford, D.B., H.W. Benjamin, and N. Kleckner. 1991. Kinetic and structural analysis of a cleaved donor intermediate and strand transfer product in Tn 10 transposition. Cell 64: 171179 .

Haykinson, M.J. and R.C. Johnson. 1993. DNA looping and the helical repeat in vitro and in vivo: Effect of $\mathrm{HU}$ protein and cnhancer location on Hin invertasome assembly. EMBO J. 12: $2503-2512$.

Huisman, O., P.R. Errada, L. Signon, and N. Kleckner. 1989. Mutational analysis of IS 10 's outside end. EMBO /. 8: 21012109.

Jain, C. and N. Kleckner. 1993. Preferential cis action of IS10 transposase depends upon its mode of synthesis. Mol. Microbiol. 9: 249-260.

Josson, K., T. Sheirlink, F. Michiels, C. Platteeuw, P. Stanssens, H. Joos, P. Dhaese, M. Zabeau, and J. Mahillon. 1989. Characterization of a Gram-positive broad host range plasmid isolated from Lactobacillus hilgardii. Plasmid 11: 53-61.

Kleckner, N. 1989. Transposon Tn10. In Mobile DNA /ed. D.E. Berg and M.M. Howcl, pp. 227-268. American Socicty for Microbiology, Washington, D.C.

- 1990a. Regulating Tn10 and IS10 transposition. Genetics 124: 449-454.

1990b. Regulation of transposition in bacteria. Annu. Rev. Cell Biol. 6: 297-327.

Kleckner, N., R.K. Chan, B.-K. Ty, and D. Botstein. 1975. Mutagenesis by insertion of a drug-resistance element carrying an inverted repetition. I. Mol. Biol. 97: 561-575.

Kleckner, N., K. Reichardt, and D. Botstein. 1979. Inversions and deletions of the Salmonella chromosome generated by the translocatable tetracycline-resistance element Tn10. $/$. Mol. Biol. 127: 89-115.

Lavoie, B.D. and G. Chaconas. 1993. Site-specific HU binding in the $\mathrm{Mu}$ transpososome: Conversion of a sequence-independent DNA-binding protein into a chemical nuclease. Genes \& Dev. 7: 2510-2519.

Lawrence, J.G., D.E. Dykhuizen, R.F. DuBose, and D.L. Hartl. 1989. Phylogenetic analysis using insertion sequence fingerprinting in Escherichia coli. Mol. Biol. Evol. 6: 1-14.

Liu, L.F. and J.C. Wang. 1987. Supercoiling of the DNA template during transcription. Proc. Natl. Acad. Sci. 84: 7024-7027.

Miller, H.I. 1984. Primary structure of the himA gene of E. coli:
Homology with DNA binding protein $\mathrm{HU}$ and association with the phenylalanyl tRNA synthetase operon. Cold Spring Harbor Symp. Quant. Biol. 49: 691-698.

Miller, J. 1972. Experiments in molecular genetics. Cold Spring Harbor Laboratory, Cold Spring Harbor, New York.

Morisato, D. and N. Kleckner. 1987. Tn10 transposition and circle formation in vitro. Cell 51: 101-111.

Morisato, D., J.C. Way, J.-H. Kim, and N. Kleckner. 1983. Tn10 transposase acts preferentially on nearby transposon ends in vivo. Cell 32: 799-807.

Mousset, S. and R. Thomas. 1969. Ter, a function which generates the ens of the mature $\lambda$ chromosome. Nature 221: 242244.

Myers, R.M., L.S. Lerman, and T. Maniatis. 1985. A general method for saturation mutagenesis of cloned DNA fragments. Science 229: 242-247.

Nash, H.A. 1990. Bending and supercoiling of DNA at the attachment site of bacteriophage $\lambda$. Trends Biochem. Sci. 15: 222-227.

Navas, I., J.M. Garcia-Loba, J. Leon, and J.M. Ortiz. 1985. Structural and functional analyses of the fosfomycin resistance transposon Tn2921. J. Bacteriol. 162: 1061-1067.

Pettijohn, D.E. and Y. Hodges-Garcia. 1990. Role of HU protein in transitional DNA coiling. In The bacterial chromosome (ed. K. Drlica and M. Riley), pp. 241-245. American Society for Microbiology, Washington, D.C.

Pontiggia, A., A. Negri, M. Beltrame, and M.E. Bianchi. 1993. Protein HU binds specifically to kinked DNA. Mol. Microbiol. 7: 343-350.

Raleigh, E.A. and N. Kleckner. 1984. Multiple IS10 rearrangements in E coli. J. Mol. Biol. 173: 437-461.

Reiss, B., R. Sprengel, and H. Schaller. 1984. Protein fusions with the kanamycin resistance gene from transposon Tn5. $E M B O$ 1. 3: 3317-3.322.

Roberts, D.E. 1986. "Genetic analysis of mutants of $E$. coli affected for Tn 10 transposition." Ph.D. thesis, Department of Biochemistry and Molecular Biology, Harvard University, Cambridge, MA.

Roberts, D., B.C. Hoopes, W.R. McClure, and N. Kleckner. 1985. IS 10 transposition is regulated by DNA adenine methylation. Cell 43: 117-130.

Roberts, D., D. Morisato, and N. Kleckner. 1987. The role of the bacterial host in the mechanism and regulation of $\mathrm{Tn} 10$ transposition. In Banbury Report 30. Eukaryotic transposable elements as mutagenic agents (ed. M.E. Lambert, J.F. McDonald, and I.B. Weinstein), pp. 17-28. Cold Spring Harbor Laboratory, Cold Spring Harbor, New York.

Roberts, T.M., S.L. Swanberg, A. Poteete, G. Riedel, and K. Backman. 1980. A plasmid cloning vehicle allowing positive selection for inserted fragments. Gene 12: 123-127.

Romero, D.A. and T.R. Klaenhammer. 1993. Transposable elements in Lactococci: A review. I. Dairy Sci. 76: 1-19.

Segall, A.M., S.D. Goodman, and H.A. Nash. 1994. Architectural elements in nucleoprotein complexes: interchangeability of specific and non-specific DNA binding proteins. EMBO I. 19: 4536-4548.

Shen, M., E.A. Raleigh, and N. Kleckner. 1987. Physical analysis of IS10-promoted transpositions and rearrangements. Genetics 116: 359-369

Simons, R.W. and N. Kleckner. 1983. Translational control of IS10 transposition. Cell 34: 683-691.

-2. 1988. Biological regulation by anti-sense RNA in prokaryotes. Annu. Rev. Genet. 22: 567-600.

Sinden, R.R., J.O. Carlson, and D.E. Pettijohn. 1980. Torsional tension in the DNA double helix measured with trimethylpsoralen in living E. coli cells: Analagous measurements in 
insect and human cells. Cell 21: 773-783.

Slater, S. and R. Maurer. 1993. Simple phagemid-based system for generating allele replacements in Escherichia coli. J. Bacteriol. 175: 4260-4262.

Stanley, J., N. Baquar, and E.J. Threlfall. 1993. Genotypes and phylogenetic relationships of Salmonella typhimurium are defined by molecular fingerprinting of IS200 and 16S rrn loci. J. Gen. Microbiol. 139: 1133-1140.

Sussman, J.K. 1992. "Escherichia coli mutations which affect expression of the IS10 transposase gene." Ph.D. thesis, Department of Microbiology, University of California at Los Angeles.

Wang, Z. and R.M. Harshey. 1994. Crucial role for DNA supercoiling in $\mathrm{Mu}$ transposition: A kinetic study. Proc. Natl. Acad. Sci. 91: 699-703.

Way, J.C. 1984. "The ends of Tn10." Ph.D. thesis, Department of Biochemistry and Molecular Biology, Harvard University, Cambridge, MA.

Werner, M.H., G.M. Clore, A.M. Gronenborn, and H.A. Nash. 1994. Symmetry and asymmetry in the function of Escherichia coli integration host factor: Implications for target identification by DNA-binding proteins. Curr. Biol. 4: 477 487.

Wolf, R.E. Jr. 1980. Integration of specialized transducing bacteriophage lambda cI857 St68 h80 dgnd his by an unusual pathway promotes formation of deletions and generates a new translocatable element. /. Bacteriol. 142: 588-602.

Yang, C.-C. and H.A. Nash. 1989. The interaction of E. coli IHF protein with its specific binding sites. Cell 57: 869-880.

Zulianello, L.E. de la Gorgue de Rosny, P. van Ulsen, P. van de Putte, and N. Goosen. 1994. The himA and himD subunits of integration host factor can specifically bind to DNA as homodimers. $E M B O$ I. 15: 1534-1540. 


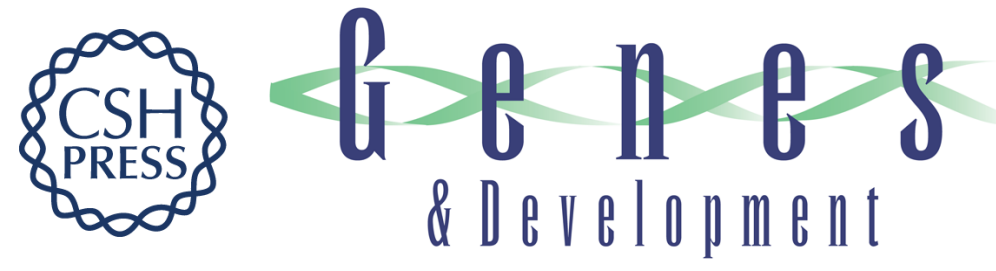

\section{Negative and positive regulation of Tn10/IS10-promoted recombination by IHF: two distinguishable processes inhibit transposition off of multicopy plasmid replicons and activate chromosomal events that favor evolution of new transposons.}

L Signon and N Kleckner

Genes Dev. 1995, 9:

Access the most recent version at doi:10.1101/gad.9.9.1123

References This article cites 56 articles, 18 of which can be accessed free at: http://genesdev.cshlp.org/content/9/9/1123.full.html\#ref-list-1

License

Email Alerting

Receive free email alerts when new articles cite this article - sign up in the box at the top Service right corner of the article or click here.

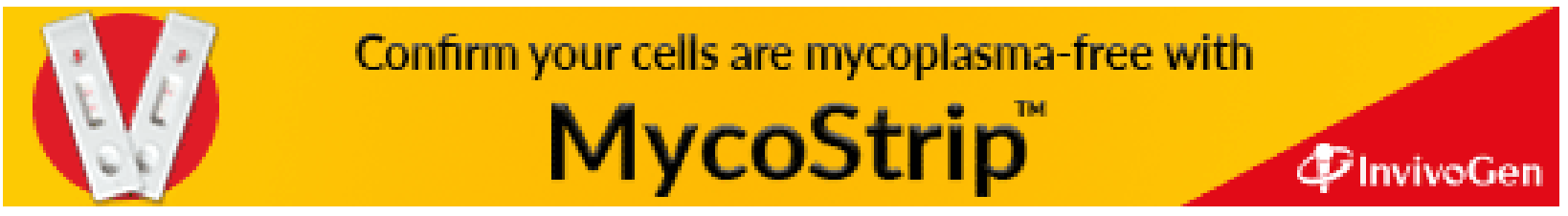

